\title{
Analysis of individual azurocidin N-glycosylation sites in regard to its secretion by insect cells, susceptibility to proteolysis and antibacterial activity
}

\author{
Katarzyna Indyk, Teresa Olczak, Justyna Ciuraszkiewicz, Wiesław Wątorek \\ and Mariusz Olczak ${ }^{凶}$ \\ Laboratory of Biochemistry, Faculty of Biotechnology, University of Wroctaw, Wroctaw, Poland
}

Received: 24 February, 2007; revised: 21 June, 2007; accepted: 06 July, 2007

available on-line: 26 July, 2007

\begin{abstract}
Azurocidin is an inactive serine protease homolog with primary sequence similarity to neutrophil elastase, cathepsin G, and proteinase 3. The aim of this study was to investigate possible consequences of differential glycosylation of azurocidin in regard to its secretion, protein stability as measured by susceptibility to proteolysis, and antibacterial activity. Site-directed mutagenesis was employed to generate mutant azurocidin variants lacking individual N-glycosylation sites. Our results show that $\mathrm{N}$-linked glycans may play a role in proper azurocidin folding and subsequent secretion by insect cells. We also demonstrate that $\mathrm{N}$-linked glycosylation contributes to azurocidin stability by protecting it from proteolysis. The lack of $\mathrm{N}$-glycosylation at individual sites does not significantly influence the azurocidin antibacterial activity.
\end{abstract}

Keywords: azurocidin, glycosylation, protein secretion, antimicrobial activity

\section{INTRODUCTION}

Azurocidin, also known as heparin-binding protein (HBP) or $37-\mathrm{kDa}$ cationic antimicrobial protein (CAP37), is an inactive serine protease homolog with the highest primary sequence similarity to neutrophil elastase and proteinase 3 (for references see Watorek, 2003). Human azurocidin lacks proteolytic activity due to substitutions (H41S and $\mathrm{S} 175 \mathrm{G}$ ) in the catalytic triad. The protein is constitutively expressed during neutrophil differentiation and targeted to azurophilic granules. Recently, the presence of azurocidin has been shown in the endothelium of Alzheimer's brain microvessels (Pereira et al., 1996a), in the endothelium associated with atherosclerotic plaques (Lee et al., 2002), in pancreatic juice (Gronborg et al., 2004), and several reports have shown that its expression is induced in endothelial (Lee et al., 2002) and corneal epithelial cells (Ruan et al., 2002) in response to inflammatory mediators such as lipopolysaccharide (LPS) or cytokines. During evolution, azurocidin adopted new functions in host defense against invading organisms by mechanisms not involving proteolytic activity. Azurocidin is a potent chemoattractant for monocytes (Pereira et al., 1990) and microglia (Pereira et al., 2003), stimulates monocyte survival and differentiation and thrombospondin secretion (Ostergaard \& Flodgaard, 1992), enhances the LPSstimulated release of prostaglandin E2 (Heinzelmann et al., 1998), interleukin-6 (Rasmussen et al., 1996), and tumor necrosis factor- $\alpha$ (Rasmussen et al., 1996; Heinzelmann et al., 1998; 1999). Azurocidin also stimulates reversible contraction of fibroblasts and endothelial cells (Ostergaard \& Flodgaard, 1992) and activates endothelial cell protein kinase C (Pereira et al., 1996b). Several studies have shown that azurocidin has a potent bactericidal activity against a variety of bacteria (Shafer et al., 1984; 1986; Gabay et al., 1989; Campanelli et al., 1990) and fungicidal activity against Candida albicans (Gabay et al., 1989; Campanelli et al., 1990).

Corresponding author: Mariusz Olczak, Laboratory of Biochemistry, Faculty of Biotechnology, University of Wroclaw, Tamka 2, 50-137 Wrocław, Poland; phone: (48 71) 3752 710; fax (48 71) 3752 608; e-mail: Mariusz.Olczak@biotech.uni.wroc.pl Abbreviations: cfu, colony-forming unit; LPS, lipopolysaccharide. 
It has been widely shown that $\mathrm{N}$-glycosylation significantly affects intracellular proteolytic processing, secretion efficiency, and posttranslational stability of secreted proteins. In human azurocidin, three N-linked glycosylation sites have been identified (Asn100, Asn114, and Asn145) (Iversen et al., 1997; 1999, Karlsen et al., 1998). The Asn114 glycosylation site is part of a putative endothelial cell protein kinase $\mathrm{C}$ activation site, thus it can be important for the cellular activation mechanism. The Asn100 and Asn114 glycosylation sites are located in a long surface loop which serves as a linker connecting two similar $\beta$-barrel domains, each composed of six antiparallel $\beta$-strands. These glycosylation sites may be involved in the folding of the two domains into the correct position with respect to each other. The Asn145 glycosylation site is situated in a $\beta$-strand in the second $\beta$-barrel (Iversen et al., 1997; Karlsen et al., 1998). The native protein isolated from neutrophils has a high variety of $\mathrm{N}$-linked glycan structures (Olczak \& Watorek, 2002), and the dominant glycans are $\mathrm{GlcNAc}_{2} \mathrm{Man}_{2}$ (Fuc), GlcNAc $\mathrm{Man}_{3}$ GlcNAc GalNeu(Fuc), and $\mathrm{GlcNAc}_{2} \mathrm{Man}$ (Fuc). The dominant glycoform found in recombinant protein produced in Sf9 insect cells contains two $\mathrm{GlNAA}_{2} \mathrm{Man}_{3}(\mathrm{Fuc})$ and one $\mathrm{GlcNAc}_{2} \mathrm{Man}_{3}$ unit (Rasmussen et al., 1996). Both native azurocidin purified from neutrophils and recombinant one produced in insect cells show high glycosylation microheterogeneity (Pereira, 1995; Rasmussen et al., 1996; Karlsen et al., 1998). The aim of this study was to investigate possible consequences of differential glycosylation of azurocidin in regard to its secretion, stability as measured by susceptibility to proteolysis, and antibacterial activity.

\section{MATERIALS AND METHODS}

Cell culture and transfection. Sf 9 TriEx insect cells (Novagen) were propagated in TriEx insect cell medium at $28^{\circ} \mathrm{C}$ as recommended by the manufacturer. Transient transfection and protein analyses were performed in 12- or 6-well plates as described previously (Olczak \& Olczak, 2006). Transfection with different amounts of wild-type DNA (pAzu1 plasmid; Table 1) was used in preliminary experiments to evaluate the influence of the amount of transfected DNA on azurocidin secretion. No significant dependence was found on the amount of plasmid DNA used (not shown), therefore for all experiments presented in this study $2 \mu \mathrm{g}$ of plasmid DNA was used. Cells and media were collected after 96 h. Before harvesting, the integrity of the cells was checked using Trypan Blue staining. The cells were confluent and viable (viability at least 95\%; data not shown). Cells were separated by centrifugation of the suspension at
$800 \times \mathrm{g}$ for $10 \mathrm{~min}$ at $4^{\circ} \mathrm{C}$. Non-concentrated medium was subjected to all analyses.

Mutagenesis. Mutagenesis was performed using the QuikChange Multi Site-Directed Mutagenesis Kit (Stratagene) with the pIEx-4 vector containing the entire azurocidin coding sequence (resulting in pAzu1 plasmid) as the template (Olczak \& Olczak, 2006). Mutations were generated using the following primers: AzuN100S (5'-GACCGTGAGGCCAGCCTCACCAGCAGC-3'), AzuN114S (5'-CTGCCTCTGCAGAGCGCCACGGTGGAAG-3'), and AzuN145S (5'-CCCAGGTTTGTCAGCGTGACTGTGACCCC-3'). After mutagenesis, the entire coding sequence of all the mutants was examined by sequencing (Institute of Biochemistry and Biophysics PAS, Warszawa, Poland). Analysis of the predicted azurocidin tertiary structure after introduction of the mutations was performed using the UCSF Chimera program (http:// www.cgl.ucsf.edu/chimera) with azurocidin models deposited in Protein Data Bank (PDB IDs: 1AE5 and 1A7S; Iversen et al., 1997; Karlsen et al., 1998). We found that neither mutation changed the overall azurocidin structure (not shown).

Purification of azurocidin. Recombinant azurocidin was purified from culture medium using cobalt-affinity chromatography as described previously (Olczak \& Olczak, 2006). For this purpose, Sf9 cells were grown in $10 \mathrm{ml}$ of TriEx medium at $28^{\circ} \mathrm{C}$ with shaking at 145 r.p.m. for 90 h. Native human azurocidin was purified from neutrophils (obtained from "buffy coats" provided by the Wroclaw District Blood Bank) according to Watorek et al. (1996) with the following modifications. Briefly, frozen granules were suspended in $50 \mathrm{mM}$ acetate buffer, $\mathrm{pH}$ 4.0 , containing $1 \mathrm{M} \mathrm{NaCl}$ and homogenized using a glass homogenizer. The granule extract was centrifuged at $30000 \times g$ for $30 \mathrm{~min}$ at $4^{\circ} \mathrm{C}$ and the pellet was re-homogenized five times. Granule extract was adjusted to $\mathrm{pH} 8.0$ using solid Tris and applied to a Sepharose-Trasylol column equilibrated in $50 \mathrm{mM}$ Tris/ $\mathrm{HCl}$ buffer, $\mathrm{pH}$ 8.0, containing $1 \mathrm{M} \mathrm{NaCl}$. After elution of non-adsorbed proteins with the equilibration buffer, human neutrophil elastase and cathepsin $\mathrm{G}$ were eluted with $50 \mathrm{mM}$ citrate buffer, $\mathrm{pH}$ 4.0, containing $1 \mathrm{M} \mathrm{NaCl}$. Then, elution of azurocidin was accomplished by eluting with $100 \mathrm{mM}$ glycine/ $\mathrm{HCl}$ buffer, $\mathrm{pH}$ 3.0. Fractions containing azurocidin were concentrated using an Amicon ultrafiltration unit (YM1).

Tryptic digestion. Culture media containing azurocidin glycosylation variants were adjusted with the medium of cells transfected with an empty vector to the same concentration of recombinant protein. Samples were incubated with trypsin (the molar trypsin:protein ratio was 1:500; Sigma) at $37^{\circ} \mathrm{C}$ in $100 \mathrm{mM}$ Tris $/ \mathrm{HCl}, \mathrm{pH} 8.0$, containing 20 $\mathrm{mM} \mathrm{CaCl}{ }_{2}$. At various time points aliquots were 
removed in which trypsin activity was stopped by adding SDS/PAGE sample buffer and immediate boiling. Controls without trypsin were included in the protocol.

Antibacterial assay. Radial diffusion assays were performed according to Lehrer et al. (1991) and McCabe et al. (2002) with the following modifications. Escherichia coli K12 Row cells (PCM 1862 strain) were grown overnight in 3\% Trypticase soy broth (TSB; Beckton Dickinson). To obtain mid-logarithmic growth phase $\left(\mathrm{OD}_{600}=0.55-0.65\right), 150 \mu \mathrm{l}$ of overnight culture was inoculated into $50 \mathrm{ml}$ of fresh TSB and incubated for additional $3-3.5 \mathrm{~h}$ at $37^{\circ} \mathrm{C}$. Bacteria were harvested by centrifugation at $2500 \times g$ for $7 \mathrm{~min}$ at $4^{\circ} \mathrm{C}$ and washed twice with cold $10 \mathrm{mM}$ sodium phosphate buffer, $\mathrm{pH}$ 7.5. Suspension containing $10^{8} \mathrm{cfu}\left(\mathrm{OD}_{620}=0.2\right.$ corresponds to $5 \times 10^{7}$ $\mathrm{cfu} / \mathrm{ml}$ ) was added to $12 \mathrm{ml}$ of warm $0.8 \%$ low electroendosmosis agarose (Sigma) in $10 \mathrm{mM}$ sodium phosphate buffer, $\mathrm{pH} 7.5$, containing $0.02 \%$ Triton X100 (Roth) and $0.02 \%$ bovine serum albumin (BSA) (Roth). After dispersing of the bacteria, the suspension was poured into 90-mm diameter Petri dishes. Several dilutions of neutrophil-derived and recombinant azurocidin were added to wells punched in the agarose layer $(7.5 \mu \mathrm{l}$ per well). The plates were incubated for $3 \mathrm{~h}$ at $37^{\circ} \mathrm{C}$ to allow protein diffusion and then overlaid with $10 \mathrm{ml}$ of $1 \%$ agar (BIOCORP) containing 6\% TSB. Following an 18-h incubation at $37^{\circ} \mathrm{C}$ the plates were stained for $24 \mathrm{~h}$ according to Lehrer et al. (1991). The areas of the clearing zones surrounding the wells were measured using a Stemi SV 11 stereomicroscope (Carl Zeiss). All assays were performed at least three times. In all experiments lysozyme (Serva) was used as a positive control. Buffer used for azurocidin dilution served as a negative control.

Bacterial membrane permeabilization assay. The assay was performed according to Mak et al. (2007) using E. coli JM83 strain harboring pCH110 plasmid (Pharmacia-Amersham) encoding $\beta$-galactosidase and ampicillin resistance, with the following modifications. Aliquots of $4 \mu \mathrm{l}$ of bacterial suspension $\left(2.5 \times 10^{8} \mathrm{cfu} / \mathrm{ml}\right)$ were mixed in wells of $96-$ well round-bottom polypropylene microtiter plates (Greiner) with $60 \mu \mathrm{l}$ of azurocidin samples in a buffer at the $\mathrm{pH}$ range 4.5-7.5. In addition, several $\mathrm{NaCl}$ concentrations $(0-0.35 \mathrm{M})$ were tested. The final amount of azurocidin was up to $2.6 \mu \mathrm{g}$ per well. The plates were incubated with shaking for $15 \mathrm{~min}$ at $37^{\circ} \mathrm{C}$. Then, $150 \mu \mathrm{l}$ of $3.3 \mathrm{mM} \beta$-galactosidase colorimetric substrate (o-nitrophenyl- $\beta$-D-galactopyranoside, ONPG; Sigma) in $0.2 \mathrm{M}$ Tris/ $\mathrm{HCl}, \mathrm{pH}$ 8.0, was added to each well and the plates were again incubated for $30 \mathrm{~min}$ at $37^{\circ} \mathrm{C}$. The reaction was stopped by adding $45 \mu \mathrm{l}$ of $1.7 \mathrm{M} \mathrm{Na}_{2} \mathrm{CO}_{3}$. The mixtures (200 $\mu$ l) were individually transferred to flat-bottom clear polyethylene 96-well plates (Nunc) and the absorbance at $420 \mathrm{~nm}$ was measured using a Spectra Max 340 microplate spectrophotometer (Molecular Devices). As control samples for $0 \%$ perforation served bacteria incubated without azurocidin, and as control samples for $100 \%$ perforation, bacteria disintegrated by sonication prior to the assay.

Protein analyses. Total protein concentration was determined using a modified Bradford method (Roti-Nanoquant, Roth) (Zor \& Selinger, 1996). Recombinant azurocidin was analyzed by SDS/PAGE and Western blotting using anti-polyHis antibodies conjugated with horseradish peroxidase (Sigma), as described previously (Olczak \& Olczak, 2006).

\section{RESULTS AND DISCUSSION}

\section{Secretion of azurocidin glycosylation variants by insect cells}

$\mathrm{N}$-glycosylation is acquired in the endoplasmic reticulum and Golgi apparatus, both of which form part of the secretory pathway. Therefore, understanding the importance of individual $\mathrm{N}$-linked sites with respect to azurocidin secretion would be advantageous. It has been shown that recombinant azurocidin expressed in baculovirus-infected insect cells possesses similar oligosaccharide structures as the native neutrophil-derived protein (Almeida et al., 1996; Rasmussen et al., 1996; Karlsen et al., 1998). Recently, we have reported on a directly transfected insect cell system suitable for analysis of multiple azurocidin variants, including glycosylation mutants (Olczak \& Olczak, 2006). We showed that recombinant azurocidin containing its native signal peptide and a fusion protein at the C-terminus was effectively expressed in transiently transfected insect cells and secreted into the culture medium at high levels (Olczak \& Olczak, 2006). In this study we used site-directed mutagenesis to remove, either singly or in combination, the $\mathrm{N}$-glycosylation sites by replacing the Asn residue from the consensus glycosylation sequon (Asn-X-Thr/ Ser) by a Ser residue (Table 1). To determine whether azurocidin affected in the glycosylation sites can be effectively secreted, the respective protein mutants were expressed in transiently transfected insect cells and analyzed by SDS/PAGE and Western blotting. A clear decrease in the molecular mass of the mutated variants of azurocidin was observed after detection with Coomassie Brilliant Blue G-250 or anti-His antibodies (Fig. 1). Each successive mutation reduced the apparent molecular mass of the recombinant mutant protein, suggesting that the mutations prevented the addition of oligosaccharides. Our results showed that secretion of single glycosylation mutants was comparable with that observed for the wild-type re- 
Table 1. Description of azurocidin mutants altered in N-glycosylation sites

\begin{tabular}{|c|c|c|c|c|c|}
\hline \multirow[t]{2}{*}{ Azurocidin variant } & \multirow[t]{2}{*}{$\begin{array}{l}\text { Expression } \\
\text { plasmid }\end{array}$} & \multicolumn{3}{|c|}{$\begin{array}{l}\text { Occupancy of N-glycosylation sites } \\
\text { by oligosaccharide chains }{ }^{\mathrm{a}}\end{array}$} & \multirow[t]{2}{*}{$\begin{array}{l}\text { Protein } \\
\text { secretion }^{b}\end{array}$} \\
\hline & & Asn100 & Asn114 & Asn145 & \\
\hline Wild-type & pAzuWT & $\bullet$ & $\bullet$ & $\bullet$ & ++++ \\
\hline N100S & pAzu1 & & $\bullet$ & - & ++++ \\
\hline N114S & pAzu2 & - & & $\bullet$ & ++++ \\
\hline N145S & pAzu3 & - & $\bullet$ & & ++++ \\
\hline N100S/N114S & pAzu1-2 & & & $\bullet$ & ++ \\
\hline N100S/N145S & pAzu1-3 & & • & & ++ \\
\hline N114S/N145S & pAzu2-3 & - & & & ++ \\
\hline N100S/N114S/N145S & pAzu1-2-3 & & & & + \\
\hline
\end{tabular}

${ }^{a}$ The presence of oligosaccharide chains $(\bullet)$ was checked experimentally by determination of electrophoretic mobility after SDS/PAGE of secreted azurocidin. ${ }^{b}$ Recombinant protein secretion was examined by Western blotting.

combinant protein, secretion of double glycosylation mutants was slightly decreased, whereas in the case of the triple glycosylation mutant the decrease in secretion was pronounced (Fig. 1). This effect could be explained by the function of Asn-linked oligosaccharides in the overall protein stability and/or folding. Iversen et al. (1999) showed that the lack of glycosylation did not affect the folding of azurocidin, as the secondary and tertiary structures of non-glycosylated recombinant azurocidin and wild-type recombinant azurocidin were nearly identical. The surface loops to which some glycans are attached were also unaffected by the removal of the glycosylation. In contrast,

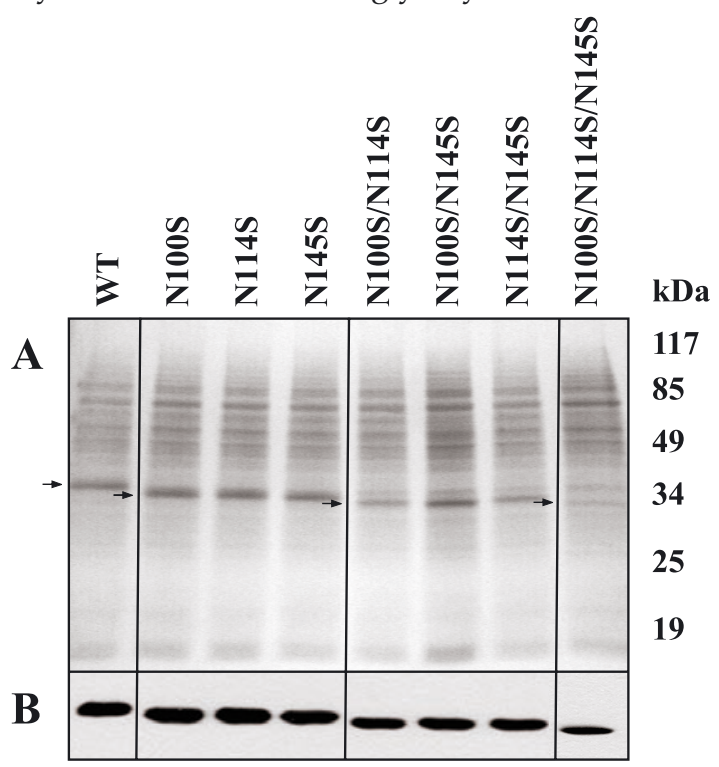

Figure 1. Secretion of recombinant azurocidin glycosylation variants by insect cells.

Protein secretion was examined by SDS/PAGE and Western blotting. Secreted azurocidin (shown by arrows) was visualized in culture media by Coomassie Brilliant G-250 (A) or anti-His antibodies (B) staining. The lack of individual N-glycans was evidenced by the decrease in the molecular mass of the mutant proteins. At least three clones were examined, for each mutation, giving similar secretion levels; the results are shown for representative clones.
Almeida et al. (1996) demonstrated that recombinant azurocidin produced in tunicamycin-treated insect cells was found exclusively in a cell-associated form, suggesting that glycosylation may be important for azurocidin stability during secretion process. In addition, all attempts to produce azurocidin in E. coli cells have failed (Almeida et al., 1996). Based on those data and our results presented in this study one may suggest that $\mathrm{N}$-linked glycans might be important for proper azurocidin folding and subsequent secretion.

\section{Susceptibility of azurocidin glycosylation variants to tryptic digestion}

It is generally accepted that glycosylation is required to stabilize the protein structure. However, results concerning the susceptibility of glycoproteins to proteolysis are not convincing (van Berkel et al., 1995; van Heen et al., 2004; Valmu et al., 2005). In this study we were interested whether N-linked glycosylation is important for azurocidin stability as measured by susceptibility to proteolysis. Recombinant azurocidin glycosylation variants were examined in culture medium without purification. Proteins were digested with trypsin, separated by SDS/ PAGE, and visualized by anti-His antibodies. The digestion was repeated three times under the same conditions giving similar patterns. As shown in Fig. 2 , the decrease of glycosylation of recombinant azurocidin was correlated with an enhanced susceptibility to degradation. These results indicated that $\mathrm{N}$-linked glycosylation does contribute to azurocidin stability by protecting it from proteolysis.

\section{Antibacterial activity of azurocidin glycosylation variants}

It has been reported that the N-terminal part of azurocidin may be important for its antimicrobial activity. A synthetic peptide corresponding to az- 


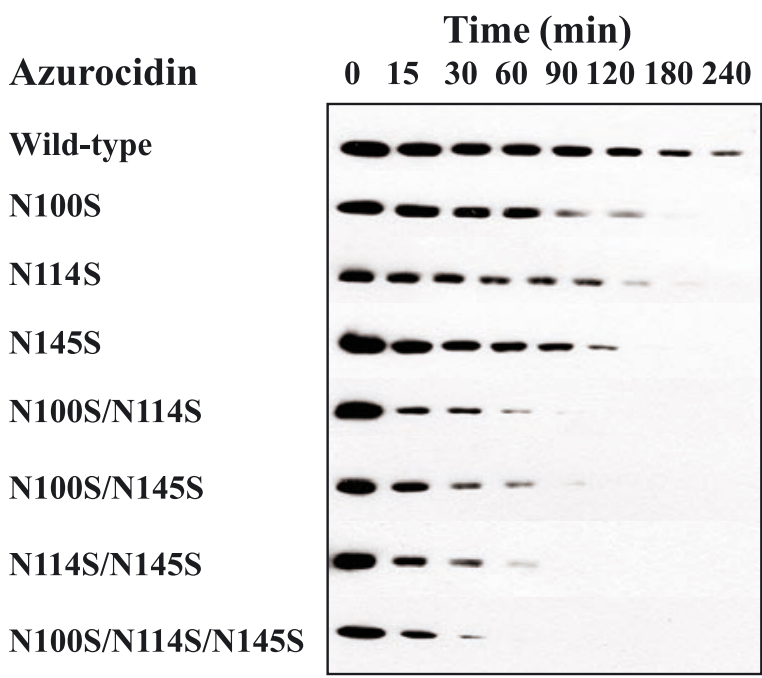

Figure 2. Susceptibility of recombinant azurocidin glycosylation variants to proteolysis.

Culture media containing azurocidin were adjusted to contain the same concentrations of the recombinant protein. The molar trypsin:protein ratio was 1:500. Samples were removed at the indicated time points and analyzed by SDS/PAGE and Western blotting and visualized with anti-His antibodies. Three independent experiments were performed; the results are shown for one representative.

urocidin amino-acid residues 20 to 44 mimicked the bactericidal activity of the protein against a range of bacteria (Pereira et al., 1993; Polikandritou et al., 1997). This peptide also exhibited a capacity to neutralize endotoxin activity in vitro and in vivo (Brackett et al., 1997). A recent report showed that binding of

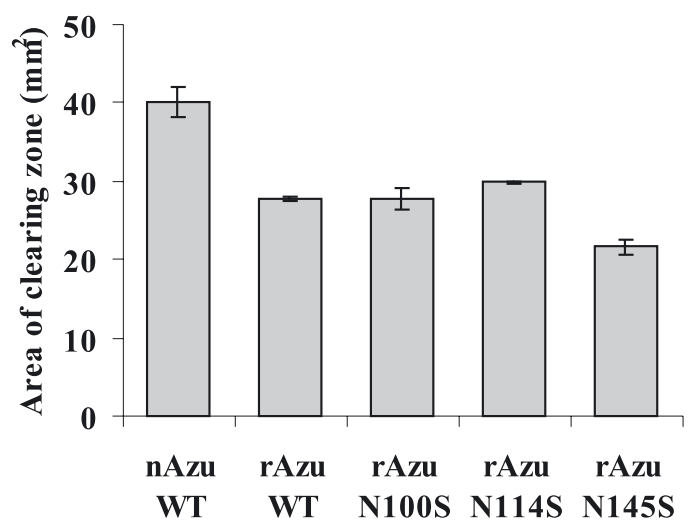

Figure 3. Antimicrobial activity of recombinant azurocidin glycosylation variants.

Antibacterial activity against E. coli was determined using radial diffusion assay. The area $\left(\mathrm{mm}^{2}\right)$ of the clearing zone surrounding the wells was measured and results are shown as means \pm S.D. for three independent experiments performed in duplicates, using $2.5 \mu \mathrm{g}$ of azurocidin per well. nAzu WT, native neutrophil-derived azurocidin; rAzu WT, recombinant wild-type azurocidin; rAzu N100S, rAzu N114S, and rAzu N145S, recombinant azurocidin glycosylation mutants. heparin, which occurred at a highly cationic region located within loops 3 and 4 of azurocidin, inhibited the azurocidin antimicrobial activity (McCabe et al., 2002). However, removal of the four positively charged amino-acid residues from the 20-44 azurocidin sequence did not affect the activity against bacteria and C. albicans (McCabe et al., 2002). Additional mutations generated within this region also did not lead to significant changes of the bactericidal activity of azurocidin (Kastrup et al., 2001).

Based on these data one may suggest that other regions or components, such as N-linked glycans, may contribute to the antibacterial function of azurocidin. To date, no reports have been published on the importance of individual N-linked glycans of human azurocidin for its antibacterial activity. To answer this question, the antibacterial activity of native neutrophil-derived and recombinant wildtype azurocidin against $E$. coli, as determined by radial diffusion assay, was first compared. The dose dependence of the activity of recombinant azurocidin was similar compared with that observed for the native neutrophil-derived protein (not shown), which is in agreement with other studies (Almeida et al., 1996). As shown in Fig. 3, neutrophil-derived azurocidin showed a higher antibacterial activity compared with wild-type recombinant azurocidin. This may be explained by the presence of the Cterminal fusion protein attached to the recombinant protein or by different purification methods used. When the results of a radial diffusion experiment performed with wild-type recombinant azurocidin were compared with those for single glycosylation mutants it was clear that only the N145S azurocidin mutant exhibited a slightly decreased antibacterial activity (Fig. 3). It has been shown previously that a complete lack of glycosylation strongly reduced the biological activity of azurocidin, since the nonglycosylated protein mediated a limited stimulation of LPS-induced cytokine release from monocytes (Iversen et al., 1999). Although the lack of N-glycosylation at individual sites did not significantly influence the antibacterial activity, all these data support the hypothesis that the oligosaccharide moiety of human azurocidin may play a role in its biological function.

Several investigators have suggested that the bacterial killing mechanism is initiated by the binding of azurocidin to bacterial LPS/lipid A (Pereira et al., 1993; Iversen et al., 1997; Brackett et al., 1997; Polikandritou et al., 1997). Upon binding to the lipid A component of LPS, azurocidin may induce leakage of the bacterial membrane. However our results showed that azurocidin, neutrophil-derived or recombinant, did not show membrane permeabilizing effect on $E$. coli cells under conditions used (not shown). 


\section{CONCLUDING REMARKS}

In this study, site-directed mutagenesis was employed to generate various azurocidin mutant proteins lacking individual N-glycosylation sites. Our results showed that although the lack of single $\mathrm{N}$-linked glycans did not influence azurocidin secretion, the absence of two or three N-linked glycans might play a role in azurocidin folding and subsequent secretion by insect cells. Azurocidin lacking $\mathrm{N}$-linked glycans, either singly or in combination, appeared to be more susceptible to trypsin digestion than its fully glycosylated form. The lack of occupancy of single N-glycosylation sites did not significantly influence the azurocidin antibacterial activity. We also found that azurocidin did not exhibit a membrane permeabilizing effect on E. coli cells. Based on the literature data and the results presented in this study it seems that further characterization of this important inflammatory mediator is likely to be critical to understanding the pathogenesis of various diseases and the design of effective therapeutics.

\section{Acknowledgements}

This work was supported by grant No. 2 P05A 11626 from the Department of Scientific Research, Ministry of Science and Higher Education (MNiSW, Poland).

E. coli K12 Row (PCM 1862 strain) was obtained from the Polish Collection of Microorganisms (Institute of Immunology and Experimental Therapy, PAS, Wrocław, Poland). E. coli JM83 was kindly provided by Adam Dubin and Pawel Mak (Jagiellonian University, Kraków, Poland). The stereomicroscope was used by courtesy of Piotr Kierzkowski (Zoological Institute, University of Wrocław, Poland).

\section{REFERENCES}

Almeida RP, Vanet A, Witko-Sarsat V, Melchior M, McCabe D, Gabay JE (1996) Azurocidin, a natural antibiotic from human neutrophils: expression, antimicrobial activity, and secretion. Protein Expr Purif 7: 355-366.

van Berkel PHC, Geerts MEJ, van Veen HA, Kooiman PM, Pieper FR, de Boer HA, Nuijens JH (1995) Glycosylated and unglycosylated human lactoferrins both bind iron and show identical affinities towards human lysozyme and bacterial lipopolysaccharide, but differ in their susceptibilities towards tryptic proteolysis. Biochem J 312: 107-114.

Brackett DJ, Lerner MR, Lacquement MA, He R, Pereira HA (1997) A synthetic lipopolisaccharide-binding peptide based on the neutrophil-derived protein CAP37 prevents endotoxin-induced responses in consious rats. Infect Immun 65: 2803-2811.

Campanelli D, Detmers PA, Nathan CF, Gabay JE (1990) Azurocidin and a homologous serine protease from neutrophils. Differential antimicrobial and proteolytic properties. J Clin Invest 85: 904-915.
Gabay JE, Scott RW, Campanelli D, Griffith J, Wilde C, Marra MN, Seeger M, Nathan CF (1989) Antibiotic proteins of human polymorphonuclear leukocytes. Proc Natl Acad Sci USA 86: 5610-5614.

Gronborg M, Bunkenborg J, Kristiansen TZ, Jensen ON, Yeo CJ, Hruban RH, Maitra A, Goggins MG, Pandey A (2004) Comprehensive proteomic analysis of human pancreatic juice. J Proteome Res 3: 1042-1055.

Iversen LF, Kastrup JS, Bjorn SE, Rasmussen PB, Wiberg FC, Flodgaard HJ, Larsen IK (1997) Structure of HBP, a multifunctional protein with a serine proteinase fold. Nat Struct Biol 4: 265-268.

Iversen LF, Kastrup JS, Bjorn SE, Wiberg FC, Larsen IK, Flodgaard HJ, Rasmussen PB (1999) Structure and function of the N-linked glycans of HBP/CAP37/azurocidin: crystal structure determination and biological characterization of nonglycosylated HBP. Protein Sci 8: 2019-2026.

Heinzelmann M, Mercer-Jones MA, Flodgaard H, Miller FN (1998) Heparin-binding protein (CAP37) is internalized in monocytes and increases LPS-induced monocyte activation. J Immunol 160: 5530-5536.

Heinzelmann M, Platz A, Flodgaard H, Polk HC Jr, Miller FN (1999) Endocytosis of heparin-binding protein (CAP37) is essential for the enhancement of lipopolysaccharide-induced TNF- $\alpha$ production in human monocytes. J Immunol 162: 4240-4245.

Karlsen S, Iversen LF, Larsen IK, Flodgaard HJ, Kastrup JS (1998) Atomic resolution structure of human HBP/ CAP37/azurocidin. Acta Cryst D54: 598-609.

Kastrup JS, Linde V, Pedersen AK, Stoffer B, Iversen LF, Larsen IK, Rasmussen PB, Flodgaard HJ, Bjorn SE (2001) Two mutants of human heparin binding protein (CAP37): toward the understanding of the nature of lipid A/LPS and BPTI binding. Proteins 42: 442-451.

Lee TD, Gonzalez ML, Kumar P, Chary-Reddy S, Grammas P, Pereira HA (2002) CAP37, a novel inflammatory mediator. Its expression in endothelial cells and localization to atherosclerotic lesions. Am J Pathol 160: 842-848.

Lehrer RI, Rosenman M, Harwig SS, Jackson R, Eisenhauer P (1991) Ultrasensitive assays for endogenous antimicrobial polypeptides. J Immunol Methods 137: 167-173.

Mak P, Siwek M, Pohl J, Dubin A (2007) Menstrual hemocidin HbB115-146 is an acidophilic antibacterial peptide potentiating the activity of human defensins, cathelicidin and lysozyme. Am J Reprod Immunol 57: 81-91.

McCabe D, Cukierman T, Gabay JE (2002) Basic residues in azurocidin/HBP contribute to both heparin binding and antimicrobial activity. J Biol Chem 277: 27477-27488.

Olczak M, Olczak T (2006) Comparison of different signal peptides for protein secretion in nonlytic insect cell system. Anal Biochem 359: 45-53.

Olczak M, Watorek W (2002) Structural analysis of N-glycans from human neutrophil azurocidin. Biochem Biophys Res Commun 293: 213-219.

Ostergaard E, Flodgaard H (1992) A neutrophil-derived proteolytic inactive elastase homologue (hHBP) mediates reversible contraction of fibroblasts and endothelial cell monolayers and stimulates monocyte survival and thrombospondin secretion. J Leukoc Biol 51: 316-323.

Pereira HA (1995) CAP37, a neutrophil-derived multifunctional inflammatory mediator. J Leukoc Biol 57: 805812.

Pereira HA, Spitznagel JK, Pohl J, Wilson DE, Morgan J, Palings I, Larrick JW (1990) CAP37, a 37 kD human neutrophil granule cationic protein shares homology with inflammatory proteinases. Life Sci 46: 189-196. 
Pereira HA, Erdem I, Pohl J, Spitznagel JK (1993) Synthetic bactericidal peptide based on CAP37: a 37-kDa human neutrophil granule-associated cationic antimicrobial protein chemotactic for monocytes. Proc Natl Acad Sci USA 90: 4733-4737.

Pereira HA, Kumar P, Grammas P (1996a) Expression of CAP37, a novel inflammatory mediator, in Alzheimer's disease. Neurobiol Aging 17: 753-759.

Pereira HA, Moore P, Grammas P (1996b) CAP37, a neutrophil granule-derived protein stimulates protein $\mathrm{ki}-$ nase $\mathrm{C}$ activity in endothelial cells. J Leukoc Biol 60: $415-422$

Pereira HA, Ruan X, Kumar P (2003) Activation of microglia: a neuroinflammatory role for CAP37. Glia 41: 64-72.

Polikandritou LM, Sheu E, Lin JS, Pereira HA (1997) Interaction of a synthetic peptide based on the neutrophilderived antimicrobial protein CAP37 with dipalmitoyl phosphatidylcholine membranes. Biochim Biophys Acta 1329: 285-290.

Rasmussen PB, Bjorn S, Hastrup S, Nielsen PF, Norris K, Thim L, Wiberg FC, Flodgaard H (1996) Characterization of recombinant human $\mathrm{HBP} / \mathrm{CAP} 37 /$ azurocidin, a pleiotropic mediator of inflammation-enhancing LPSinduced cytokine release from monocytes. FEBS Lett 390: 109-112.

Ruan X, Chodosh J, Callegan MC, Booth MC, Lee TD, Kumar P, Gilmore MS, Pereira HA (2002) Corneal expression of the inflammatory mediator CAP37. Invest Ophthalmol Vis Sci 43: 1414-1421.
Shafer WM, Martin LE, Spitznagel JK (1984) Cationic antimicrobial proteins isolated from human neutrophil granulocytes in the presence of diisopropyl fluorophosphate. Infect Immun 45: 29-35.

Shafer WM, Martin LE, Spitznagel JK (1986) Late intraphagosomal hydrogen ion concentration favors the in vitro antimicrobial capacity of a 37-kilodalton cationic granule protein of human neutrophil granulocytes. Infect Immun 53: 651-655.

Valmu L, Kalkkinen N, Husa A, Rye PD (2005) Differential susceptibility of transferrin glycoforms to chymotrypsin: a proteomics approach to the detection of carbohydrate-deficient transferrin. Biochemistry 44: 1600716013.

van Veen HA, Geerts MEJ, van Berkel PHC, Nuijens JH (2004) The role of N-linked glycosylation in the protection of human and bovine lactoferrin against tryptic proteolysis. Eur J Biochem 271: 678-684.

Watorek W (2003) Azurocidin - inactive serine proteinase homolog acting as a multifunctional inflammatory mediator. Acta Biochim Polon 50: 743-752.

Watorek W, Polanowski A, Wilusz T (1996) The use of sequential affinity chromatography for separation of human neutrophil elastase, cathepsin $G$ and azurocidin. Acta Biochim Polon 43: 503-506.

Zor T, Selinger Z (1996) Linearization of the Bradford protein assay increases its sensitivity: theoretical and experimental studies. Anal Biochem 236: 302-308. 